\title{
THE DAUGAVET PROPERTY IN REARRANGEMENT INVARIANT SPACES
}

\author{
M. D. ACOSTA, A. KAMIŃSKA, AND M. MASTYŁO
}

\begin{abstract}
We study rearrangement invariant spaces with the Daugavet property. The main result of this paper states that under mild assumptions the only nonseparable rearrangement invariant space $X$ over an atomless finite measure space with the Daugavet property is $L_{\infty}$ endowed with its canonical norm. We also prove that a uniformly monotone rearrangement invariant space over an infinite atomless measure space with the Daugavet property is isometric to $L_{1}$. As an application we obtain that an Orlicz space over an atomless measure space has the Daugavet property if and only if it is isometrically isomorphic to $L_{1}$.
\end{abstract}

\section{INTRODUCTION}

This paper continues the investigation of ideas developed in [2] and [8]. A Banach space $X$ is said to have the Daugavet property $(X \in(D P)$ for short) if for every rank one operator $T: X \rightarrow X$, the Daugavet equation

$$
\|I+T\|=1+\|T\|
$$

is satisfied. In this case, it is known that the equation is satisfied for every weakly compact operator [9, Theorem 2.3].

Daugavet showed in 1963 that every compact operator on $C[0,1]$ satisfies the above equation [5]. Since then the Daugavet property has been studied by many authors in various contexts. We refer to a survey paper of Werner [16] as well as to the textbook [1] and the references therein. Notice that in the setting of the classical function spaces it is well known that $C(K)$ satisfies the Daugavet property when $K$ is a perfect compact topological space. Moreover, if $\mu$ is a $\sigma$-finite atomless measure, then $L_{1}(\mu)$ and $L_{\infty}(\mu)$ also have the Daugavet property. It is also well known that the disk algebra $H^{\infty}$ possesses this property. Recently the class of function spaces with the Daugavet property has been extended to spaces of Lipschitz functions on a metric space 7 .

The Daugavet property is quite restrictive in both the isomorphic and isometric sense. For instance, a Banach space with the Daugavet property does not embed into a space with an unconditional basis [9]. Some other examples of spaces with the Daugavet property are presented in $[2,9,16,17$.

Received by the editors November 29, 2012 and, in revised form, March 9, 2013.

2010 Mathematics Subject Classification. Primary 46B20, 46E30.

Key words and phrases. Daugavet property, rearrangement invariant spaces, Orlicz spaces, uniform monotonicity.

The first author was supported by MTM2012-31755, Junta de Andalucía FQM-4911 and FQM185.

The third author was supported by the National Science Centre (NCN), Poland, grant no. 2011/01/B/ST1/06243. 
The aim of this paper is to study rearrangement invariant spaces with the Daugavet property. This kind of study was initiated in the joint paper of the authors in [2], where it was shown that the Daugavet property is quite restrictive in the class of Banach function lattices. In particular, it was proved in [2, Proposition 1.6] that if an order continuous rearrangement invariant (r.i. for short) space $E$ on finite atomless measure space with the Fatou property has the Daugavet property, then $E$ coincides with $L_{1}$ up to equivalence of norms. Further, in [8] it was proved that the only separable real r.i. space on $[0,1]$ with the Daugavet property is $L_{1}[0,1]$ endowed with its canonical norm. It is then natural to ask whether a nonseparable r.i. space with the Daugavet property is necessarily isometric to the space $L_{\infty}$ equipped with the standard norm. We address this problem in Section 3. Our main result of this section (Theorem 3.4) in particular gives a positive answer for that mentioned question in the case of an r.i. space over a finite atomless measure space.

In Section 4 we show under mild assumptions that an r.i. space on an atomless infinite measure space is isometrically isomorphic to $L_{1}$ whenever it has the Daugavet property. In particular, the characterization holds for uniformly monotone spaces as well as for arbitrary Orlicz spaces. In connection with our results we state the conjecture that the only r.i. spaces on infinite atomless measure spaces with the Daugavet property are those which are naturally isometric either to $L_{1}$ or $L_{\infty}$ endowed with their canonical norms. We finish the paper with a few remarks and some open problems.

\section{Preliminaries}

We will use the standard notation from Banach space theory. If $X$ is a Banach space we denote by $B_{X}$ and $S_{X}$ the closed unit ball and the unit sphere of $X$, respectively. Throughout the paper we will consider only real Banach spaces.

Let $(\Omega, \mathcal{S}, \mu)$ denote a complete and $\sigma$-finite measure space. As usual $L^{0}:=$ $L^{0}(\Omega, \mathcal{S}, \mu)$ denotes the space of all equivalence classes of real-valued measurable functions on $\Omega$. A Banach (function) lattice $\left(E,\|\cdot\|_{E}\right)$ on $(\Omega, \mathcal{S}, \mu)$ is defined to be a subspace of $L^{0}$, endowed with a complete norm $\|\cdot\|_{E}$ and such that there exists $u \in E$ with $u>0$ a.e., and if $x \in L^{0}$ satisfies $|x| \leq|y|$ a.e., for some $y \in E$, then $x \in E$ and $\|x\|_{E} \leq\|y\|_{E}$.

Let $E$ be a Banach lattice on $(\Omega, \mathcal{S}, \mu)$. An element $x \in E$ is called order continuous if for every sequence $\left(x_{n}\right)$ in $E$ such that $0 \leq x_{n} \leq|x|$ and $x_{n} \downarrow 0$ a.e., it holds that $\left\|x_{n}\right\|_{E} \rightarrow 0$. The subspace of $E$ consisting of all order continuous elements from $E$ is denoted by $E_{a}$. Then $E$ is said to be order continuous provided that $E_{a}=E$. It is said that $E$ has the Fatou property (or $E$ is maximal) if for any $x \in L^{0}, x_{n} \in E$ such that $0 \leq x_{n} \leq x, x_{n} \uparrow x$ a.e., and $\sup \left\|x_{n}\right\|_{E}<\infty$ we have that $x \in E$ and $\left\|x_{n}\right\|_{E} \rightarrow\|x\|_{E}$. We say that $E$ has the weak Fatou property whenever if $x_{n}, x \in E, 0 \leq x_{n} \leq x, x_{n} \uparrow x$ a.e., then $\left\|x_{n}\right\|_{E} \rightarrow\|x\|_{E}$. It is clear that order continuous Banach lattices have the weak Fatou property.

The Köthe dual space (or associate space) $E^{\prime}$ of a Banach function lattice $E$ on $(\Omega, \mathcal{S}, \mu)$ is defined as the space of all $x \in L^{0}$ such that $\int_{\Omega}|x y| d \mu<\infty$ for every $y \in E$. It is a Banach function lattice on $(\Omega, \mathcal{S}, \mu)$ when equipped with the (dual) norm

$$
\|x\|_{E^{\prime}}=\sup _{\|y\|_{E} \leq 1} \int_{\Omega}|x y| d \mu .
$$


It is well known that a Banach lattice $E$ is order continuous if and only if the map $E^{\prime} \ni y \mapsto x_{y}^{*} \in E^{*}$ given by

$$
x_{y}^{*}(x)=\int_{\Omega} x y d \mu, \quad x \in E,
$$

is an order isometric isomorphism of $E^{\prime}$ onto $E^{*}$ (see, e.g., [1] ). Let us also remark that the Köthe dual space $E^{\prime}$ is quite often identified with a subspace of the so-called integral functionals in $E^{*}$, and the identification is given by the above formula.

We notice that any Köthe dual $E^{\prime}$ of $E$ satisfies the Fatou property as well as a number of classical spaces such as Lebesgue spaces $L_{p}, 1 \leq p \leq \infty$, Orlicz spaces or more general Musielak-Orlicz or Nakano spaces.

A Banach lattice $E$ is said to be a $K B$-space (Kantorovich-Banach space) whenever every positive increasing norm bounded sequence of $E$ is norm convergent, that is, $E$ is order continuous and has the Fatou property.

In what follows we will use the following observations without any references. Let $E$ be a Banach lattice on $(\Omega, \mathcal{S}, \mu)$. If $\operatorname{supp}\left(E_{a}\right)=\Omega$, then it easy to see that $\left(E_{a}\right)^{\prime}=E^{\prime}$ with equality of norms, and so

$$
\left(E_{a}\right)^{\prime \prime}=\left(\left(E_{a}\right)^{\prime}\right)^{\prime}=\left(E^{\prime}\right)^{\prime}=E^{\prime \prime} .
$$

Since $E_{a} \hookrightarrow E \hookrightarrow E^{\prime \prime}$ with the norm of the inclusion maps less than or equal to 1 , it follows by the above equalities that $E_{a}$ has the Fatou property if and only if $E_{a}=E=E^{\prime \prime}$ with equality of norms.

Thus we conclude that if $E$ is not order continuous and $\operatorname{supp}\left(E_{a}\right)=\Omega$, then $E_{a}$ has the weak Fatou property; however it is not maximal, i.e., it does not have the Fatou property.

An important class of Banach lattices are rearrangement invariant (r.i. for short) spaces. We recall its definition below. Given $x \in L^{0}$, its distribution function is defined by $\mu_{x}(\lambda)=\mu\{t \in \Omega:|x(t)|>\lambda\}, \lambda \geq 0$. Then a Banach lattice $E$ on $(\Omega, \mathcal{S}, \mu)$ is an r.i. space whenever $y \in E$ and $\|x\|_{E}=\|y\|_{E}$ whenever $\mu_{x}=\mu_{y}$ and $x \in E$. It is well known [3, 12] that for any r.i. space $E$ we have

$$
L_{1} \cap L_{\infty} \subset E \subset L_{1}+L_{\infty}
$$

and so in particular this implies that any element $x \in E$ is locally integrable. Clearly, for an r.i. space $E,\left\|\chi_{A}\right\|_{E}$ depends only on $\mu(A)$ for any measurable set $A$ of finite measure. If $(\Omega, \mathcal{S}, \mu)$ is an atomless measure space, then for every $t \in \mathbb{R}_{+}$ with $t \leq \mu(\Omega)$, we may define the function $\phi_{E}$ by $\phi_{E}(t)=\left\|\chi_{A}\right\|_{E}$, where $A$ is any measurable set with $\mu(A)=t$. This function is called the fundamental function of $E$. For every r.i. space $E$ on an atomless measure space $(\Omega, \mathcal{S}, \mu)$ we have that

$$
\phi_{E}(t) \phi_{E^{\prime}}(t)=t, \quad 0 \leq t<\mu(\Omega)
$$

(see for instance [3, 12]). As a consequence, we get the following inequality.

Lemma 2.1. For any r.i. space $E$ on an atomless measure space $(\Omega, \mathcal{S}, \mu)$ and every $A \in \mathcal{S}$ with $0<\mu(A)<\infty$ and $\mu(A) \leq \mu(\Omega)$, it is satisfied that

$$
\frac{\phi_{E}(\mu(A))}{\mu(A)} \int_{A}|x| d \mu \leq\left\|x \chi_{A}\right\|_{E}, \quad x \in E .
$$

In the sequel we will need a somewhat technical lemma. For the sake of completeness we include a proof. In what follows we will denote by $L_{1}$ or $L_{\infty}$ the spaces $L_{1}(\Omega, \mathcal{S}, \mu)$ or $L_{\infty}(\Omega, \mathcal{S}, \mu)$, respectively. 
Lemma 2.2. For any r.i. space $E$ on an atomless measure space $(\Omega, \mathcal{S}, \mu)$ we have:

(i) If $\phi_{E}(t) \leq t$ for every $t \in \mathbb{R}_{+}$with $t \leq \mu(\Omega)$, then the inclusion map id: $L_{1} \rightarrow E$ is continuous with $\|\mathrm{id}\| \leq 1$.

(ii) If $\phi_{E}(t) \geq t$ for every $t \in \mathbb{R}_{+}$with $t \leq \mu(\Omega)$, then the inclusion map id: $E \rightarrow L_{1}$ is continuous with $\|\mathrm{id}\| \leq 1$.

(iii) $E=L_{1}$ with equality of norms if and only if $\phi_{E}(t)=t$ for all $t \in \mathbb{R}_{+}$with $t<\mu(\Omega)$.

Proof. (i) Let $x=\sum_{i=1}^{n} a_{i} \chi_{A_{i}}$ be a simple function in $L_{1}$ with $A_{i} \in \mathcal{S}$ for every $i$, $A_{i} \cap A_{j}=\emptyset$ for $i \neq j$, and $a_{i} \in \mathbb{R}$ for each $1 \leq i \leq n$. Then we have

$$
\|x\|_{E} \leq \sum_{i=1}^{n}\left|a_{i}\right|\left\|\chi_{A_{i}}\right\|_{E}=\sum_{i=1}^{n}\left|a_{i}\right| \phi_{E}\left(\mu\left(A_{i}\right)\right) \leq \sum_{i=1}^{n}\left|a_{i}\right| \mu\left(A_{i}\right)=\|x\|_{1} .
$$

Now, if $x \in L_{1}$ is arbitrary, then we can find a sequence $\left(x_{n}\right)$ of simple functions such that

$$
\left\|x_{n}-x\right\|_{1} \rightarrow 0
$$

In particular, this implies that $\left(x_{n}\right)$ is a Cauchy sequence in $L_{1}$. From the inequality shown above, we obtain that $\left(x_{n}\right)$ is also a Cauchy sequence in $E$, and so $x_{n} \rightarrow y$ in $E$ for some $y \in E$. By continuous embedding $E \hookrightarrow L^{0}$ we have

$$
x_{n} \rightarrow y \quad \text { in } L^{0} .
$$

Since also $x_{n} \rightarrow x$ in $L^{0}, x=y$, and so

$$
\|x\|_{E}=\lim _{n \rightarrow \infty}\left\|x_{n}\right\|_{E} \leq \lim _{n \rightarrow \infty}\left\|x_{n}\right\|_{1}=\|x\|_{1},
$$

which completes the proof of (i).

(ii) Our hypothesis on $\phi_{E}$ implies that $\phi_{E^{\prime}}(t) \leq 1$ for every real number $t$ with $0<t \leq \mu(\Omega)$. Thus for any simple function $x=\sum_{i=1}^{n} a_{i} \chi_{A_{i}}$ in $L_{\infty}$ expressed in its canonical form, we obtain

$$
\|x\|_{E^{\prime}} \leq \max _{1 \leq i \leq n}\left|a_{i}\right|\left\|\sum_{i=1}^{n} \chi_{A_{i}}\right\|_{E^{\prime}}=\max _{1 \leq i \leq n}\left|a_{i}\right| \phi_{E^{\prime}}\left(\mu\left(\bigcup_{i=1}^{n} A_{i}\right)\right)=\|x\|_{\infty} .
$$

Similarly as in the proof of (i) this yields (by the density of simple functions in $L_{\infty}$ ) that

$$
\|x\|_{E^{\prime}} \leq\|x\|_{\infty}, \quad x \in L_{\infty} .
$$

In consequence by Köthe duality we obtain that $\|x\|_{1} \leq\|x\|_{E^{\prime \prime}}$ for all $x \in E^{\prime \prime}$. Since id: $E \rightarrow E^{\prime \prime}$ with $\|$ id $\| \leq 1$, we get the required statement.

(iii) This statement is an immediate consequence of (i) and (ii).

Before we state a result that will be used later we recall that an r.i. space $E$ is called fully symmetric (or fully r.i.) whenever it is an exact interpolation space between $L_{1}$ and $L_{\infty}$; i.e., for any linear operator $T: L_{1}+L_{\infty} \rightarrow L_{1}+L_{\infty}$ such that $\|T\|_{L_{1} \rightarrow L_{1}} \leq 1$ and $\|T\|_{L_{\infty} \rightarrow L_{\infty}} \leq 1$, we have that $T$ maps $E$ into $E$ and $\|T\|_{E \rightarrow E} \leq 1$.

It is well known [3,12 that maximal as well as order continuous r.i. spaces are fully symmetric. 
Lemma 2.3. Let $E$ be a fully symmetric space on $(\Omega, \mathcal{S}, \mu)$. Let $\Omega=\Omega_{1} \cup \Omega_{2}$, where $\Omega_{i}$ are disjoint and measurable for $i=1,2$ with $0<\mu\left(\Omega_{2}\right)<\infty$. Then for any $x \in E$ it is satisfied that

$$
\left\|x \chi_{\Omega_{1}}+\left(\frac{1}{\mu\left(\Omega_{2}\right)} \int_{\Omega_{2}} x\right) \chi_{\Omega_{2}}\right\|_{E} \leq\|x\|_{E} .
$$

Proof. Let $T$ be the linear operator on $L_{1}+L_{\infty}$ defined by

$$
T x=x \chi_{\Omega_{1}}+\left(\frac{1}{\mu\left(\Omega_{2}\right)} \int_{\Omega_{2}} x\right) \chi_{\Omega_{2}}, \quad x \in L_{1}+L_{\infty} .
$$

It is easy to see that $T: L_{1} \rightarrow L_{1}$ and $T: L_{\infty} \rightarrow L_{\infty}$ with $\|T\|_{L_{1} \rightarrow L_{1}} \leq 1$ and $\|T\|_{L_{\infty} \rightarrow L_{\infty}} \leq 1$. Since $E$ is fully symmetric, $\|T x\|_{E} \leq\|x\|_{E}$ for all $x \in E$, and so this yields the required inequality.

We recall the well-known characterization of the Daugavet property given in [9. Lemma 2.2].

Lemma 2.4. Let $X$ be a Banach space. The following conditions are equivalent:

(i) The space $X$ has the Daugavet property.

(ii) For every $x \in S_{X}, y^{*} \in S_{X^{*}}$ and $\varepsilon>0$ there is $x^{*} \in S_{X^{*}}$ such that $x^{*}(x)>1-\varepsilon$ and $\left\|x^{*}+y^{*}\right\|>2-\varepsilon$.

(iii) For every $x \in S_{X}, x^{*} \in S_{X^{*}}$ and $\varepsilon>0$ there is $y \in S_{X}$ such that $x^{*}(y)>$ $1-\varepsilon$ and $\|x+y\|>2-\varepsilon$.

\section{The Daugavet Property in R.I. SPACES ISOMorphic to $L_{\infty}$}

In 2 the Daugavet property was studied in Banach lattices on atomless $\sigma$-finite measure spaces. It was proved [2, Proposition 1.6] that any order continuous r.i. space on a finite atomless measure space with the Fatou property, which has the Daugavet property, must coincide as a set with $L_{1}$ and the norms are equivalent. Further in [8] it has been shown that the only separable r.i. space on $[0,1]$ with the Daugavet property is $L_{1}[0,1]$ endowed with its canonical norm.

It is well known that $L_{\infty}[0,1]$ endowed with its canonical norm has the Daugavet property. Similarly as in the case of $L_{1}[0,1]$, a natural question arises whether a nonseparable r.i. space over $[0,1]$ with the Daugavet property must coincide with $L_{\infty}[0,1]$ equipped with its standard norm. This question was left out unsolved in [8]. In this section we provide some general results which in particular answers the above question in the positive.

We need some preliminary results. The first one stated below is a variant of the well-known characterization of the Daugavet property [9, Lemma 2.2].

Lemma 3.1. Let $X$ be a Banach space and $B \subset S_{X^{*}}$ a subset satisfying $-B \subset B$ and 1-norming, i.e.,

$$
\|x\|=\sup _{b^{*} \in B}\left|b^{*}(x)\right| \quad \text { for all } x \in X .
$$

Then $X$ has the Daugavet property if and only if for every $x_{0} \in S_{X}, x_{0}^{*} \in S_{X^{*}}$, and $\varepsilon>0$ there is a $b^{*} \in B$ such that

$$
b^{*}\left(x_{0}\right)>1-\varepsilon \quad \text { and } \quad\left\|x_{0}^{*}+b^{*}\right\|>2-\varepsilon .
$$


Proof. Sufficiency is immediate by Lemma 2.4. Conversely, assume that $X$ has the Daugavet property. Given $x_{0} \in S_{X}, x_{0}^{*} \in S_{X^{*}}$ and $\varepsilon>0$ we consider the following rank-one operator $T: X \rightarrow X$ given by

$$
T(x)=x_{0}^{*}(x) x_{0}, \quad x \in X .
$$

Since $\|T\|=1$ and $X \in(D P),\|I+T\|=1+\|T\|=2$. Hence, there is $x \in S_{X}$ so that

$$
\|x+T x\|>2-\frac{\varepsilon}{2} \text {. }
$$

We can clearly assume (by using $-x$ instead of $x$ if necessary) that $x_{0}^{*}(x) \geq 0$. Assume that $0<\varepsilon<1$. Since $B \subset S_{X^{*}}$ is 1-norming and $-B \subset B$, then there is $b^{*} \in B$ so that

Hence

$$
b^{*}(x+T x)=b^{*}(x+T x)>2-\frac{\varepsilon}{2} .
$$

$$
2-\frac{\varepsilon}{2}<b^{*}(x+T x)=b^{*}(x)+b^{*}(T x) \leq b^{*}(x)+1 .
$$

We deduce that

$$
1-\frac{\varepsilon}{2}<b^{*}(x)
$$

By repeating the same argument and by using the fact that $x_{0}^{*}(x) \geq 0$ we obtain

$$
1-\frac{\varepsilon}{2}<b^{*}(T x)=x_{0}^{*}(x) b^{*}\left(x_{0}\right) \leq \min \left\{x_{0}^{*}(x), b^{*}\left(x_{0}\right)\right\} .
$$

Finally, we have that $b^{*}\left(x_{0}\right)>1-\varepsilon$ and

$$
\left\|b^{*}+x_{0}^{*}\right\| \geq\left(b^{*}+x_{0}^{*}\right)(x)>2-\varepsilon,
$$

which completes the proof.

Notice that for every Banach space $X$, the set $\operatorname{ext}\left(B_{X^{*}}\right)$ of the extreme points of $B_{X^{*}}$ is a 1-norming set. The following simple example shows that the assumption of the condition in the above lemma only for elements $x_{0}^{*} \in B$ does not imply that $X$ satisfies the Daugavet property.

Example 3.2. The space $X=\ell_{1}$ does not satisfy the Daugavet property. However, it satisfies the following condition for $B=\operatorname{ext}\left(B_{X^{*}}\right)$ : for every $x_{0} \in S_{X}, x_{0}^{*} \in B$, and $\varepsilon>0$ there is a functional $b^{*} \in B$ such that

$$
b^{*}\left(x_{0}\right)>1-\varepsilon \quad \text { and } \quad\left\|x_{0}^{*}+b^{*}\right\|>2-\varepsilon .
$$

To see this take $x_{0} \in S_{X}, b_{0}^{*} \in \operatorname{ext}\left(B_{X^{*}}\right)$. Since $X^{*}=\ell_{\infty}$ isometrically via the usual identification, we have

$$
\sum_{n=1}^{\infty}\left|x_{0}(n)\right|=1, \text { and }\left|b_{0}^{*}(n)\right|=1 \text { for all } n \in \mathbb{N} .
$$

Let $\left(e_{n}\right)$ be the unit vector basis of $\ell_{1}$. Given $\varepsilon>0$, since $x_{0} \in \ell_{1}$, we can find $N$ large enough such that if $P_{N}(x):=\sum_{k=1}^{N} x(k) e_{k}$ for $x=(x(k)) \in \ell_{1}$, then

$$
\sum_{k=1}^{N}\left|x_{0}(k)\right|=\left\|P_{N}\left(x_{0}\right)\right\|>1-\frac{\varepsilon}{2} \Rightarrow\left\|\left(I-P_{N}\right)\left(x_{0}\right)\right\|<\frac{\varepsilon}{2} .
$$

Now if we take the element $b^{*} \in \ell_{\infty}$ given by

$$
b^{*}(k)=\operatorname{sign} x_{0}(k), \quad 1 \leq k \leq N,
$$


and

$$
b^{*}(N+1)=\operatorname{sign} b_{0}^{*}(N+1), \quad b^{*}(k)=1, \quad k \geq N+2,
$$

it is clear that $b^{*} \in \operatorname{ext}\left(B_{\ell_{\infty}}\right)=\operatorname{ext}\left(B_{X^{*}}\right)$. By the choice of $b^{*}$ and $N$ we obtain that

$$
\begin{aligned}
b^{*}\left(x_{0}\right) & =\sum_{k=1}^{\infty} b^{*}(k) x_{0}(k)=\sum_{k=1}^{N} b^{*}(k) x_{0}(k)+\sum_{k=N+1}^{\infty} b^{*}(k) x_{0}(k) \\
& =\sum_{k=1}^{N}\left|x_{0}(k)\right|+b^{*}\left(\left(I-P_{N}\right)\left(x_{0}\right)\right)>1-\frac{\varepsilon}{2}-\left\|b^{*}\right\|\left\|\left(I-P_{N}\right)\left(x_{0}\right)\right\| \\
& \geq 1-\frac{\varepsilon}{2}-\left\|\left(I-P_{N}\right)\left(x_{0}\right)\right\|=1-\varepsilon .
\end{aligned}
$$

We also have that

$$
\left\|b_{0}^{*}+b^{*}\right\| \geq\left|\left(b_{0}^{*}+b^{*}\right)(N+1)\right|=2 .
$$

So we checked the stated condition for every $x_{0}^{*} \in B=\operatorname{ext}\left(B_{X^{*}}\right)$.

Remark 3.3. It follows by the reversed Krein-Milman Theorem that for any Banach space $X$ and every symmetric 1 -norming subset $B \subset B_{X^{*}}$ we have $\operatorname{ext}\left(B_{X^{*}}\right) \subset \bar{B}^{w^{*}}$, where $\bar{B}^{w^{*}}$ is the closure of $B$ in the $w^{*}$-topology in $X^{*}$. Thus we conclude that if $X=\ell_{1}$, then for any $B \subset B_{X^{*}}$ symmetric and 1-norming subset, and for every $n$ and for every choice of signs $\left\{\varepsilon_{1}, \ldots, \varepsilon_{n}\right\}$ there is an element $b^{*} \in B$ such that $\left|\varepsilon_{i}-b^{*}(i)\right|<\varepsilon$ for every $1 \leq i \leq N$.

So for $X=\ell_{1}$, by using the above argument, the condition stated in Lemma 3.1 holds for every 1-norming subset $B \subset B_{X^{*}}$ and for every $x_{0}^{*} \in B$. However, $\ell_{1}$ does not have the Daugavet property.

Inspired by [8] we are ready to prove the main result of this section.

Theorem 3.4. Let $E$ be an r.i. space over atomless finite measure space $(\Omega, \mathcal{S}, \mu)$ with the weak Fatou property. If $\phi_{E^{\prime}}(0+)=\lim _{t \rightarrow 0+} \phi_{E^{\prime}}(t)=0$ and $E$ has the Daugavet property, then $E$ is isometrically isomorphic to $L_{\infty}$. Moreover, the isometry is given by an appropriate multiple of the formal identity between both spaces.

Proof. Without loss of generality we can assume that $\mu(\Omega)=1$ and $\phi_{E}(1)=1$. Under this assumption we will prove that the identity map is an isometry from $L_{\infty}$ onto $E$. First observe that by Lemma 2.1 we have the inclusions

$$
E \hookrightarrow L_{1} \quad \text { and } \quad E^{\prime} \hookrightarrow L_{1},
$$

and both operators have norm one.

The element $x_{0}:=-\chi_{\Omega}$ satisfies $x_{0} \in S_{E}$, and so the integral functional $x_{0}^{*}$ generated by $\chi_{\Omega}$ is in $S_{E^{*}}$ since

$$
\left\|x_{0}\right\|_{E}=\phi_{E}(1)=1, \quad\left\|x_{0}^{*}\right\|_{E^{*}}=\phi_{E^{\prime}}(1)=1 .
$$

Since $E$ has the weak Fatou property, $E^{\prime}$ is a 1-norming subspace of the dual space $E^{*}$ (see, e.g., [15, Proposition 1.b.18]). By assumption, $X$ has the Daugavet property, and so in view of Lemma 3.1 for every $\varepsilon>0$ there is an integral functional $x_{\varepsilon}^{*} \in S_{E^{*}}$ such that

a) $x_{\varepsilon}^{*}\left(x_{0}\right)>1-\varepsilon$,

b) $\left\|1+x_{\varepsilon}^{*}\right\|_{E^{*}}>2-\varepsilon$. 
Since $x_{\varepsilon}^{*} \in S_{E^{*}}$ is an integral functional for every $\varepsilon>0$, there exists $f_{\varepsilon} \in S_{E^{\prime}}$ such that

$$
x_{\varepsilon}^{*}(x)=\int_{\Omega} f_{\varepsilon} x d \mu, \quad \forall x \in E .
$$

Now, let us consider the following measurable subsets of $\Omega$ :

$$
P_{\varepsilon}=\left\{t \in \Omega: f_{\varepsilon}(t) \geq 0\right\}, \quad N_{\varepsilon}=\left\{t \in \Omega: f_{\varepsilon}(t)<0\right\} .
$$

We have $P_{\varepsilon}=P_{\varepsilon}^{1} \cup P_{\varepsilon}^{2}$ and $N_{\varepsilon}=N_{\varepsilon}^{1} \cup N_{\varepsilon}^{2}$, where

$$
\begin{array}{cl}
P_{\varepsilon}^{1}=\left\{t \in P_{\varepsilon}: f_{\varepsilon}(t) \leq 2\right\}, & P_{\varepsilon}^{2}=\left\{t \in P_{\varepsilon}: f_{\varepsilon}(t)>2\right\}, \\
N_{\varepsilon}^{1}=\left\{t \in N_{\varepsilon}:-2 \leq f_{\varepsilon}(t)\right\}, & N_{\varepsilon}^{2}=\left\{t \in N_{\varepsilon}: f_{\varepsilon}(t)<-2\right\} .
\end{array}
$$

By condition a) we have that

$$
\begin{aligned}
1-\varepsilon<x_{\varepsilon}^{*}\left(x_{0}\right) & =-\int_{\Omega} f_{\varepsilon} d \mu=-\int_{P_{\varepsilon}} f_{\varepsilon} d \mu-\int_{N_{\varepsilon}} f_{\varepsilon} d \mu \\
& \leq-\int_{N_{\varepsilon}} f_{\varepsilon} d \mu=\int_{N_{\varepsilon}}\left|f_{\varepsilon}\right| d \mu \leq\left\|f_{\varepsilon}\right\|_{1} \leq 1 .
\end{aligned}
$$

As a consequence,

$$
\left\|f_{\varepsilon} \chi_{P_{\varepsilon}}\right\|_{1}=\int_{P_{\varepsilon}}\left|f_{\varepsilon}\right| d \mu=\left\|f_{\varepsilon}\right\|_{1}-\int_{N_{\varepsilon}}\left|f_{\varepsilon}\right| d \mu \leq 1-\int_{N_{\varepsilon}}\left|f_{\varepsilon}\right| d \mu \leq \varepsilon,
$$

and so

$$
\int_{P_{\varepsilon}^{1}} f_{\varepsilon} d \mu \leq \varepsilon
$$

Clearly $0 \leq f_{\varepsilon} \chi_{P_{\varepsilon}^{1}} \leq 2$, and so the family of functions $\left\{f_{\varepsilon} \chi_{P_{\varepsilon}^{1}}: \varepsilon>0\right\}$ is bounded in $L_{\infty}$. In view of (3.2) we obtain $\lim _{\varepsilon \rightarrow 0}\left\|f_{\varepsilon} \chi_{P_{\varepsilon}^{1}}\right\|_{1}=0$, and hence $f_{\varepsilon} \chi_{P_{\varepsilon}^{1}} \rightarrow 0$ in measure as $\varepsilon \rightarrow 0$. Since $\phi_{E^{\prime}}(0+)=0$, we deduce

$$
\lim _{\varepsilon \rightarrow 0}\left\|f_{\varepsilon} \chi_{P_{\varepsilon}^{1}}\right\|_{E^{\prime}}=0 .
$$

Further $f_{\varepsilon}>2$ on $P_{\varepsilon}^{2}$ implies by (3.2) that

$$
2 \mu\left(P_{\varepsilon}^{2}\right) \leq \int_{P_{\varepsilon}^{2}} f_{\varepsilon} d \mu \leq \int_{P_{\varepsilon}} f_{\varepsilon} d \mu<\varepsilon,
$$

and thus

$$
\mu\left(P_{\varepsilon}^{2}\right)<\frac{\varepsilon}{2}
$$

Let

$$
\Delta_{\varepsilon}^{1}=P_{\varepsilon}^{1} \cup N_{\varepsilon}^{1}, \quad \Delta_{\varepsilon}^{2}=P_{\varepsilon}^{2} \cup N_{\varepsilon}^{2} .
$$

Since id: $E^{\prime} \rightarrow L_{1}$ with $\|$ id $\| \leq 1$,

$$
2 \mu\left(\Delta_{\varepsilon}^{2}\right) \leq \int_{P_{\varepsilon}^{2} \cup N_{\varepsilon}^{2}}\left|f_{\varepsilon}\right| d \mu \leq\left\|x_{\varepsilon}^{*}\right\|_{E^{*}}=1,
$$

and so

$$
\mu\left(\Delta_{\varepsilon}^{2}\right) \leq 1 / 2 .
$$

Since $-2 \leq f_{\varepsilon}<0$ on $N_{\varepsilon}^{1}$ and $f_{\varepsilon} \leq-2$ on $N_{\varepsilon}^{2}$, we obtain

$$
\left|\chi_{N_{\varepsilon}^{1}}+f_{\varepsilon} \chi_{N_{\varepsilon}^{1}}\right| \leq \chi_{N_{\varepsilon}^{1}}, \quad\left|\chi_{N_{\varepsilon}^{2}}+f_{\varepsilon} \chi_{N_{\varepsilon}^{2}}\right| \leq\left|f_{\varepsilon}\right| \chi_{N_{\varepsilon}^{2}} .
$$


Hence in view of (3.7) we get

$$
\begin{aligned}
\left|1+f_{\varepsilon}\right| & =\left|\left(\chi_{N_{\varepsilon}^{1}}+f_{\varepsilon} \chi_{N_{\varepsilon}^{1}}\right)+\left(\chi_{N_{\varepsilon}^{2}}+f_{\varepsilon} \chi_{N_{\varepsilon}^{2}}\right)+\left(\chi_{P_{\varepsilon}^{1}}+f_{\varepsilon} \chi_{P_{\varepsilon}^{1}}\right)+\left(\chi_{P_{\varepsilon}^{2}}+f_{\varepsilon} \chi_{P_{\varepsilon}^{2}}\right)\right| \\
& \leq \chi_{N_{\varepsilon}^{1}}+\left|f_{\varepsilon}\right| \chi_{N_{\varepsilon}^{2}}+\left(\chi_{P_{\varepsilon}^{1}}+\left|f_{\varepsilon}\right| \chi_{P_{\varepsilon}^{1}}\right)+\left(\chi_{P_{\varepsilon}^{2}}+\left|f_{\varepsilon}\right| \chi_{P_{\varepsilon}^{2}}\right) \\
& =\chi_{\Delta_{\varepsilon}^{1}}+\left|f_{\varepsilon}\right| \chi_{\Delta_{\varepsilon}^{2}}+\chi_{P_{\varepsilon}^{2}}+\left|f_{\varepsilon}\right| \chi_{P_{\varepsilon}^{1}} .
\end{aligned}
$$

By condition b), combining the above estimates with (3.5) yields

$$
\begin{aligned}
2-\varepsilon \leq\left\|1+x_{\varepsilon}^{*}\right\|_{E^{*}} & =\left\|1+f_{\varepsilon}\right\|_{E^{\prime}} \leq\left\|\chi_{\Delta_{\varepsilon}^{1}}+\left|f_{\varepsilon}\right| \chi_{\Delta_{\varepsilon}^{2}}+\chi_{P_{\varepsilon}^{2}}+\left|f_{\varepsilon}\right| \chi_{P_{\varepsilon}^{1}}\right\|_{E^{\prime}} \\
& \leq\left\|\chi_{\Delta_{\varepsilon}^{1}}+f_{\varepsilon} \chi_{\Delta_{\varepsilon}^{2}}\right\|_{E^{\prime}}+\phi_{E^{\prime}}(\varepsilon / 2)+\left\|f_{\varepsilon} \chi_{P_{\varepsilon}^{1}}\right\|_{E^{\prime}} \\
& \leq \phi_{E^{\prime}}\left(\mu\left(\Delta_{\varepsilon}^{1}\right)\right)+\left\|f_{\varepsilon} \chi_{\Delta_{\varepsilon}^{2}}\right\|_{E^{\prime}}+\phi_{E^{\prime}}(\varepsilon / 2)+\left\|f_{\varepsilon} \chi_{P_{\varepsilon}^{1}}\right\|_{E^{\prime}} \\
& \leq 2+\phi_{E^{\prime}}(\varepsilon / 2)+\left\|f_{\varepsilon} \chi_{P_{\varepsilon}^{1}}\right\|_{E^{\prime}}
\end{aligned}
$$

where we used the fact that $\phi_{E^{\prime}}\left(\mu\left(\Delta_{\varepsilon}^{1}\right)\right) \leq \phi_{E^{\prime}}(1)=1,\left\|f_{\varepsilon} \chi_{\Delta_{\varepsilon}^{2}}\right\|_{E^{\prime}} \leq\left\|f_{\varepsilon}\right\|_{E^{\prime}}=1$ and $\phi_{E^{\prime}}(0+)=0$.

As a consequence of the previous remarks and (3.4), passing to the limit in (3.8) as $\varepsilon \rightarrow 0$, we deduce that

$$
\lim _{\varepsilon \rightarrow 0} \phi_{E^{\prime}}\left(\mu\left(\Delta_{\varepsilon}^{1}\right)\right)=\lim _{\varepsilon \rightarrow 0}\left\|f_{\varepsilon} \chi_{\Delta_{\varepsilon}^{2}}\right\|_{E^{\prime}}=1
$$

From (3.6) it follows that

$$
\mu\left(\Delta_{\varepsilon}^{1}\right) \geq 1 / 2 .
$$

By applying Lemma 2.1 we obtain that

$$
\frac{1}{\mu\left(\Delta_{\varepsilon}^{1}\right)}\left(\int_{\Delta_{\varepsilon}^{1}}\left|f_{\varepsilon}\right| d \mu\right) \phi_{E^{\prime}}\left(\mu\left(\Delta_{\varepsilon}^{1}\right)\right)=\frac{1}{\mu\left(\Delta_{\varepsilon}^{1}\right)}\left(\int_{\Delta_{\varepsilon}^{1}}\left|f_{\varepsilon}\right| d \mu\right)\left\|\chi_{\Delta_{\varepsilon}^{1}}\right\|_{E^{\prime}} \leq\left\|f_{\varepsilon}\right\|_{E^{\prime}}=1 .
$$

From the previous inequality and (3.9) we deduce that

$$
\limsup _{\varepsilon \rightarrow 0} \frac{1}{\mu\left(\Delta_{\varepsilon}^{1}\right)} \int_{\Delta_{\varepsilon}^{1}}\left|f_{\varepsilon}\right| d \mu \leq 1
$$

For $\varepsilon>0$, we set

$$
g_{\varepsilon}=\left|f_{\varepsilon}\right|+\left(1-\frac{1}{\mu\left(\Delta_{\varepsilon}^{1}\right)} \int_{\Delta_{\varepsilon}^{1}}\left|f_{\varepsilon}\right| d \mu\right) \chi_{\Delta_{\varepsilon}^{1}}
$$

It is clear that

$$
\left|g_{\varepsilon}\right| \chi_{\Delta_{\varepsilon}^{2}}=\left|f_{\varepsilon}\right| \chi_{\Delta_{\varepsilon}^{2}}
$$

Since

$$
\frac{1}{\mu\left(\Delta_{\varepsilon}^{1}\right)} \int_{\Delta_{\varepsilon}^{1}} g_{\varepsilon} d \mu=\frac{1}{\mu\left(\Delta_{\varepsilon}^{1}\right)} \int_{\Delta_{\varepsilon}^{1}}\left|f_{\varepsilon}\right| d \mu+1-\frac{1}{\mu\left(\Delta_{\varepsilon}^{1}\right)} \int_{\Delta_{\varepsilon}^{1}}\left|f_{\varepsilon}\right| d \mu=1,
$$

we have

$$
\left|g_{\varepsilon}\right| \chi_{\Delta_{\varepsilon}^{2}}+\left(\frac{1}{\mu\left(\Delta_{\varepsilon}^{1}\right)} \int_{\Delta_{\varepsilon}^{1}} g_{\varepsilon} d \mu\right) \chi_{\Delta_{\varepsilon}^{1}}=\left|f_{\varepsilon}\right| \chi_{\Delta_{\varepsilon}^{2}}+\chi_{\Delta_{\varepsilon}^{1}}
$$


Since $E^{\prime}$ has the Fatou property, (3.8) combined with Lemma 2.3 yields

$$
\begin{aligned}
2-\varepsilon & \leq\left\|1+x_{\varepsilon}^{*}\right\|_{E^{*}}=\left\|1+f_{\varepsilon}\right\|_{E^{\prime}} \leq\left\|\chi_{\Delta_{\varepsilon}^{1}}+f_{\varepsilon} \chi_{\Delta_{\varepsilon}^{2}}\right\|_{E^{\prime}}+\phi_{E^{\prime}}(\varepsilon / 2)+\left\|f_{\varepsilon} \chi_{P_{\varepsilon}^{1}}\right\|_{E^{\prime}} \\
& =\left\|\left|g_{\varepsilon}\right| \chi_{\Delta_{\varepsilon}^{2}}+\left(\frac{1}{\mu\left(\Delta_{\varepsilon}^{1}\right)} \int_{\Delta_{\varepsilon}^{1}} g_{\varepsilon} d \mu\right) \chi_{\Delta_{\varepsilon}^{1}}\right\|_{E^{\prime}}+\phi_{E^{\prime}}(\varepsilon / 2)+\left\|f_{\varepsilon} \chi_{P_{\varepsilon}^{1}}\right\|_{E^{\prime}} \\
& \leq\left\|g_{\varepsilon}\right\|_{E^{\prime}}+\phi_{E^{\prime}}(\varepsilon / 2)+\left\|f_{\varepsilon} \chi_{P_{\varepsilon}^{1}}\right\|_{E^{\prime}} \\
& \leq\left\|f_{\varepsilon}\right\|_{E^{\prime}}+\left|1-\frac{1}{\mu\left(\Delta_{\varepsilon}^{1}\right)} \int_{\Delta_{\varepsilon}^{1}}\right| f_{\varepsilon}|d \mu|\left\|\chi_{\Delta_{\varepsilon}^{1}}\right\|_{E^{\prime}}+\phi_{E^{\prime}}(\varepsilon / 2)+\left\|f_{\varepsilon} \chi_{P_{\varepsilon}^{1}}\right\|_{E^{\prime}} \\
& =1+\phi_{E^{\prime}}\left(\mu\left(\Delta_{\varepsilon}^{1}\right)\right)\left|1-\frac{1}{\mu\left(\Delta_{\varepsilon}^{1}\right)} \int_{\Delta_{\varepsilon}^{1}}\right| f_{\varepsilon}|d \mu|+\phi_{E^{\prime}}(\varepsilon / 2)+\left\|f_{\varepsilon} \chi_{P_{\varepsilon}^{1}}\right\|_{E^{\prime}} \\
& \leq 1+\left|1-\frac{1}{\mu\left(\Delta_{\varepsilon}^{1}\right)} \int_{\Delta_{\varepsilon}^{1}}\right| f_{\varepsilon}|d \mu|+\phi_{E^{\prime}}(\varepsilon / 2)+\left\|f_{\varepsilon} \chi_{P_{\varepsilon}^{1}}\right\|_{E^{\prime}} .
\end{aligned}
$$

Using the order continuity of $\left(E^{\prime}\right)_{a},(\underline{3.12})$ and (3.4) we deduce that

$$
\lim _{\varepsilon \rightarrow 0} \frac{1}{\mu\left(\Delta_{\varepsilon}^{1}\right)} \int_{\Delta_{\varepsilon}^{1}}\left|f_{\varepsilon}\right| d \mu=0
$$

Since $0<\mu\left(\Delta_{\varepsilon}^{1}\right) \leq 1$, we obtain

$$
\lim _{\varepsilon \rightarrow 0} \int_{\Delta_{\varepsilon}^{1}}\left|f_{\varepsilon}\right|=0,
$$

and hence $\lim _{\varepsilon \rightarrow 0}\left\|f_{\varepsilon} \chi_{N_{\varepsilon}^{1}}\right\|_{1}=0$. In view of (3.1) we know that $\lim _{\varepsilon \rightarrow 0}\left\|f_{\varepsilon} \chi_{N_{\varepsilon}}\right\|_{1}=1$ and $\left\|f_{\varepsilon}\right\|_{1} \leq\left\|f_{\varepsilon}\right\|_{E^{\prime}}=1$. Hence

$$
\lim _{\varepsilon \rightarrow 0}\left\|f_{\varepsilon} \chi_{N_{\varepsilon}^{2}}\right\|_{1}=1
$$

As a consequence, $\mu\left(N_{\varepsilon}^{2}\right)>0$ for $\varepsilon>0$ small enough, and thus by Lemma 2.1.

$$
\frac{\phi_{E^{\prime}}\left(\mu\left(N_{\varepsilon}^{2}\right)\right)}{\mu\left(N_{\varepsilon}^{2}\right)}\left\|f_{\varepsilon} \chi_{N_{\varepsilon}^{2}}\right\|_{1} \leq\left\|f_{\varepsilon} \chi_{N_{\varepsilon}^{2}}\right\|_{E^{\prime}} \leq 1
$$

In view of (3.15) we deduce that

$$
\limsup _{\varepsilon \rightarrow 0} \frac{\phi_{E^{\prime}}\left(\mu\left(N_{\varepsilon}^{2}\right)\right)}{\mu\left(N_{\varepsilon}^{2}\right)} \leq 1 .
$$

By using the fact that the function $t \mapsto \varphi(t):=\frac{\phi_{E^{\prime}}(t)}{t}$ is decreasing, $\varphi(1)=1$ and inequality (3.10), that is, $0<\mu\left(N_{\varepsilon}^{2}\right) \leq \mu\left(\Delta_{\varepsilon}^{2}\right) \leq \mu\left(\Delta_{\varepsilon}^{1}\right) \leq 1$, from (3.17) we deduce

$$
\limsup _{\varepsilon \rightarrow 0} \frac{\phi_{E^{\prime}}\left(\mu\left(\Delta_{\varepsilon}^{1}\right)\right)}{\mu\left(\Delta_{\varepsilon}^{1}\right)}=\limsup _{\varepsilon \rightarrow 0} \frac{\phi_{E^{\prime}}\left(\mu\left(N_{\varepsilon}^{2}\right)\right)}{\mu\left(N_{\varepsilon}^{2}\right)}=1 .
$$

In view of (3.9) we deduce

$$
\limsup _{\varepsilon \rightarrow 0} \frac{1}{\mu\left(\Delta_{\varepsilon}^{1}\right)}=1
$$

that is, $\lim _{\varepsilon \rightarrow 0} \mu\left(\Delta_{\varepsilon}^{1}\right)=1$. As a consequence,

$$
\lim _{\varepsilon \rightarrow 0} \mu\left(N_{\varepsilon}^{2}\right) \leq \lim _{\varepsilon \rightarrow 0} \mu\left(\Delta_{\varepsilon}^{2}\right) \leq 1-\lim _{\varepsilon \rightarrow 0} \mu\left(\Delta_{\varepsilon}^{1}\right)=0 .
$$

In view of (3.17), by using again that $\varphi$ is decreasing, we obtain that $\varphi(t)=1$ for every $0<t \leq 1$, so $\phi_{E^{\prime}}(t)=t$ for every $0<t \leq 1$ and $\phi_{E}(t)=1$. As a consequence, $E$ is isometric to $L_{\infty}$ and the formal identity from $E$ into $L_{\infty}$ is an isometry, and so the claim is proved. 
Corollary 3.5. If $E$ is an r.i. space on $[0,1]$ with the Daugavet property isomorphic to $C(K)$-space, then $E$ is isometrically isomorphic to $L_{\infty}$.

Proof. Since $C(K)$ has the Dunford-Pettis property, it follows from [10, Theorem $2]$ that $E=L_{\infty}[0,1]$ up to equivalence of norms. Thus Theorem 3.4 applies.

We can state now the following result which gives a complete description of r.i. spaces on finite atomless measure spaces with both the weak Fatou and the Daugavet properties.

Theorem 3.6. Let $E$ be an r.i. space on a finite atomless measure space $(\Omega, \mathcal{S}, \mu)$ with the weak Fatou property. If $E$ has the Daugavet property, then either $E=L_{1}$ or $E=L_{\infty}$ isometrically.

Proof. If $\operatorname{supp} E_{a}=\Omega$, then $E_{a}$ has the Daugavet property by [2, Theorem 2.1]. Since $E_{a}$ has order continuous norm the proof of Corollary 4.9 in $\left[8\right.$ ] gives $E_{a}=L_{1}$ isometrically. This implies that $E=L_{1}$ isometrically.

In the case when $E_{a}=\{0\}$ we have $\phi_{E}(0+)>0$. Hence $E=L_{\infty}$, and so $E^{\prime}=L_{1}$ up to equivalence of norms. Then $\phi_{E^{\prime}}(0+)=0$ yields by Theorem 3.4 that $E=L_{\infty}$ isometrically, and this completes the proof.

\section{Daugavet Property in Banach FUnCtion Lattices OVER INFINITE MEASURE SPACE}

In this section we investigate the Daugavet property of a Banach function lattice $E$ over infinite atomless measure space. We will show under certain geometric assumptions on $E$ that $E$ is isometric to $L_{1}$ whenever $E$ has the Daugavet property. As a consequence, $L_{1}$ is the only r.i. space whose norm is uniformly monotone that has the Daugavet property.

If $E$ is an r.i. space over a measure space $(\Omega, \mathcal{S}, \mu)$ and $A \in \mathcal{S}$, we denote $E(A)=\left\{\left.x\right|_{A}: x \in E\right\}$, where $\left.x\right|_{A}$ is the restriction of $x$ to $A$. It is clear that $\left\|\left.x\right|_{A}\right\|_{E(A)}=\left\|x \chi_{A}\right\|_{E}$.

Based on some ideas from [8] we first prove the following result.

Theorem 4.1. Let $E$ be an r.i. space over infinite atomless measure space $(\Omega, \mathcal{S}, \mu)$. Assume $E$ has the following properties:

(i) $E$ is a fully symmetric space.

(ii) For any $A \in \mathcal{S}$ with $\mu(A)<\infty$, the space $E(A)$ is order continuous.

(iii) Let $A \in \mathcal{S}$ with $\mu(A)<\infty$. If $x \in S_{E}$ and for every $\varepsilon>0, y_{\varepsilon} \in S_{E}$, then $\lim _{\varepsilon \rightarrow 0}\left\|x+y_{\varepsilon} \chi_{A}\right\|_{E}=2$ whenever $\lim _{\varepsilon \rightarrow 0}\left\|y_{\varepsilon} \chi_{A}\right\|_{E}=1$ and $\lim _{\varepsilon \rightarrow 0}\left\|x+y_{\varepsilon}\right\|_{E}=2$.

If $E$ has the Daugavet property, then it is isometrically isomorphic to $L_{1}$. In fact, if $\phi_{E}(1)=1$, then $E=L_{1}$ with equality of norms.

Proof. We will apply Lemma 2.4 (iii). Let $\varepsilon>0$ and $0<a<\mu(\Omega)$. Since $\mu$ is atomless there exists $A \in \mathcal{S}$ such that $\mu(A)=a$. Throughout the proof let $\phi(t)=\phi_{E}(t), 0 \leq t<\mu(\Omega)$. Assume without loss of generality that $\phi(1)=1$.

Choose $x=c \chi_{A}$ with $c=1 / \phi(a)$ and $y=-b \chi_{A}$ with $b=1 / \phi_{E^{\prime}}(a)=\phi(a) / a$. Then $x \in S_{E}$ and $y \in S_{E^{\prime}}$, which induces an integral functional $F \in E^{*}$ such that $F(z)=\int_{\Omega} z y d \mu$ for $z \in E$. By the Daugavet property and Lemma 2.4, there exists $y_{\varepsilon} \in S_{E}$ such that

$$
F\left(y_{\varepsilon}\right) \geq 1-\varepsilon \quad \text { and } \quad\left\|x+y_{\varepsilon}\right\|_{E}>2-\varepsilon .
$$


It follows that

$$
1-\varepsilon \leq-b \int_{A} y_{\varepsilon} d \mu \leq \int_{\Omega}\left|y_{\varepsilon} \chi_{A}\right| b \chi_{A} d \mu \leq\left\|y_{\varepsilon} \chi_{A}\right\|_{E}\left\|b \chi_{A}\right\|_{E^{\prime}} \leq 1 .
$$

Hence $1 \geq\left\|y_{\varepsilon}-y_{\varepsilon} \chi_{A^{c}}\right\|_{E}=\left\|y_{\varepsilon} \chi_{A}\right\|_{E} \geq 1-\varepsilon$. Therefore by assumption (iii) without loss of generality we can suppose that for any $\varepsilon>0$,

$$
2-\varepsilon<\left\|x+y_{\varepsilon} \chi_{A}\right\|_{E} .
$$

Let $C=\left\{s \in A: y_{\varepsilon}(s)<0\right\}$ and $D=\left\{s \in A: y_{\varepsilon}(s) \geq 0\right\}$. Also define $C_{1}=\left\{s \in C:\left|y_{\varepsilon}(s)\right| \leq 2 / \phi(a)\right\}, C_{2}=\left\{s \in C:\left|y_{\varepsilon}(s)\right|>2 / \phi(a)\right\}, D_{1}=\{s \in D:$ $\left.\left|y_{\varepsilon}(s)\right| \leq 2 / \phi(a)\right\}, D_{2}=\left\{s \in D:\left|y_{\varepsilon}(s)\right|>2 / \phi(a)\right\}$. Notice that these sets depend on $\varepsilon$.

By (4.1) we have $b \int_{A} y_{\varepsilon} d \mu=b \int_{C} y_{\varepsilon} d \mu+b \int_{D} y_{\varepsilon} d \mu \leq-1+\varepsilon$ and $b \int_{A}\left|y_{\varepsilon}\right| d \mu=$ $-b \int_{C} y_{\varepsilon} d \mu+b \int_{D} y_{\varepsilon} d \mu \leq 1$. Hence

$$
b \int_{D} y_{\varepsilon} d \mu \leq \varepsilon
$$

It follows that $\left\|y_{\varepsilon} \chi_{D_{1}}\right\|_{L_{1}(A)}=\int_{D_{1}} y_{\varepsilon} d \mu \rightarrow 0$ as $\varepsilon \rightarrow 0$, and so $y_{\varepsilon} \chi_{D_{1}} \rightarrow 0$ in measure on $A$. Moreover, $\left|y_{\varepsilon} \chi_{D_{1}}\right| \chi_{A} \leq \frac{2}{\phi(a)} \chi_{A}$, where $\frac{2}{\phi(a)} \chi_{A} \in E$ since $\mu(A)<\infty$. Now by order continuity of $E(A)$ we have that

$$
u(\varepsilon)=\left\|y_{\varepsilon} \chi_{D_{1}}\right\|_{E} \rightarrow 0, \text { as } \varepsilon \rightarrow 0 .
$$

On the other hand, by (4.3),$\varepsilon / 2 \geq \int_{D_{2}} b\left|y_{\varepsilon}\right| d \mu \geq \frac{2}{\phi(a)} \mu\left(D_{2}\right) \frac{\phi(a)}{a}=\frac{2}{a} \mu\left(D_{2}\right)$. Moreover, $\phi(\varepsilon) \rightarrow 0$ as $\varepsilon \rightarrow 0$ by order continuity of $E(A)$. Hence

$$
v(\varepsilon)=\left\|\frac{1}{\phi(a)} \chi_{D_{2}}\right\|_{E}=\frac{\phi\left(\mu\left(D_{2}\right)\right)}{\phi(a)} \leq \frac{\phi(a \varepsilon / 4)}{\phi(a)} \rightarrow 0, \text { as } \varepsilon \rightarrow 0 .
$$

We also have

$$
\frac{2}{a} \mu\left(C_{2} \cup D_{2}\right)=\frac{\phi(a)}{a} \cdot \frac{2}{\phi(a)} \mu\left(C_{2} \cup D_{2}\right) \leq b \int_{C_{2} \cup D_{2}}\left|y_{\varepsilon}\right| d \mu \leq b \int_{A}\left|y_{\varepsilon}\right| d \mu \leq 1,
$$

which implies that

$$
\mu\left(C_{2} \cup D_{2}\right) \leq a / 2 .
$$

By definition of $C_{1}$ and $C_{2}$ we have that $\left|c \chi_{C_{1}}+y_{\varepsilon} \chi_{C_{1}}\right| \leq c \chi_{C_{1}}$ and $\left|c \chi_{C_{2}}+y_{\varepsilon} \chi_{C_{2}}\right| \leq$ $\left|y_{\varepsilon}\right| \chi_{C_{2}}$. Then setting $\Delta_{i}=C_{i} \cup D_{i}$ for $i=1,2$, we obtain

$$
\begin{aligned}
\left\|c \chi_{A}+y_{\varepsilon} \chi_{A}\right\|_{E} \leq & \|\left|c \chi_{C_{1}}+y_{\varepsilon} \chi_{C_{1}}\right|+\left|c \chi_{C_{2}}+y_{\varepsilon} \chi_{C_{2}}\right| \\
& +c \chi_{D_{1}}+c \chi_{D_{2}}+\left|y_{\varepsilon}\right| \chi_{D_{1}}+\left|y_{\varepsilon}\right| \chi_{D_{2}} \|_{E} \\
\leq & \left\|c \chi_{C_{1}}+\left|y_{\varepsilon}\right| \chi_{C_{2}}+c \chi_{D_{1}}+c \chi_{D_{2}}+\left|y_{\varepsilon}\right| \chi_{D_{1}}+\left|y_{\varepsilon}\right| \chi_{D_{2}}\right\|_{E} \\
= & \left\|\left(c \chi_{\Delta_{1}}+\left|y_{\varepsilon}\right| \chi_{\Delta_{2}}\right)+\left|y_{\varepsilon}\right| \chi_{D_{1}}+c \chi_{D_{2}}\right\|_{E} \\
\leq & \left\|c \chi_{\Delta_{1}}+\left|y_{\varepsilon}\right| \chi_{\Delta_{2}}\right\|_{E}+\left\|y_{\varepsilon} \chi_{D_{1}}\right\|_{E}+\left\|c \chi_{D_{2}}\right\|_{E} \\
= & \left\|c \chi_{\Delta_{1}}+\left|y_{\varepsilon}\right| \chi_{\Delta_{2}}\right\|_{E}+u(\varepsilon)+v(\varepsilon) .
\end{aligned}
$$

Hence by (4.2),

$$
2-\varepsilon<\left\|x+y_{\varepsilon} \chi_{A}\right\|_{E} \leq\left\|c \chi_{\Delta_{1}}+\left|y_{\varepsilon}\right| \chi_{\Delta_{2}}\right\|_{E}+u(\varepsilon)+v(\varepsilon) .
$$


Defining $z_{\varepsilon}=\left|y_{\varepsilon}\right| \chi_{A}+\left(c-\frac{1}{\mu\left(\Delta_{1}\right)} \int_{\Delta_{1}}\left|y_{\varepsilon}\right| d \mu\right) \chi_{\Delta_{1}}$, it is clear that

$$
\left|z_{\varepsilon}\right| \chi_{\Delta_{2}}+\left(\frac{1}{\mu\left(\Delta_{1}\right)} \int_{\Delta_{1}}\left|z_{\varepsilon}\right| d \mu\right) \chi_{\Delta_{1}}=c \chi_{\Delta_{1}}+\left|y_{\varepsilon}\right| \chi_{\Delta_{2}} .
$$

In view of assumption (i), we can apply Lemma 2.3 and obtain

$$
\left\|\left|z_{\varepsilon}\right| \chi_{\Delta_{2}}+\left(\frac{1}{\mu\left(\Delta_{1}\right)} \int_{\Delta_{1}}\left|z_{\varepsilon}\right| d \mu\right) \chi_{\Delta_{1}}\right\|_{E} \leq\left\|z_{\varepsilon}\right\|_{E},
$$

and thus by (4.5),

$2-\varepsilon<\left\|z_{\varepsilon}\right\|_{E}+u(\varepsilon)+v(\varepsilon) \leq\left\|y_{\varepsilon}\right\|_{E}+\left|c-\frac{1}{\mu\left(\Delta_{1}\right)} \int_{\Delta_{1}}\right| y_{\varepsilon}|d \mu| \phi\left(\mu\left(\Delta_{1}\right)\right)+u(\varepsilon)+v(\varepsilon)$.

Assume that $c \leq \frac{1}{\mu\left(\Delta_{1}\right)} \int_{\Delta_{1}}\left|y_{\varepsilon}\right| d \mu$ for $\varepsilon$ small enough. Notice that by (4.4) we get $\mu\left(\Delta_{1}\right) \geq a / 2$, and so in view of Lemma 2.3 we get

$$
\begin{aligned}
&\left(\frac{1}{\mu\left(\Delta_{1}\right)} \int_{\Delta_{1}}\left|y_{\varepsilon}\right| d \mu-c\right) \phi\left(\mu\left(\Delta_{1}\right)\right)=\frac{\phi\left(\mu\left(\Delta_{1}\right)\right)}{\mu\left(\Delta_{1}\right)} \int_{\Delta_{1}}\left|y_{\varepsilon}\right| d \mu-\frac{\phi\left(\mu\left(\Delta_{1}\right)\right)}{\phi(a)} \\
& \leq\left\|\left(\frac{1}{\mu\left(\Delta_{1}\right)} \int_{\Delta_{1}}\left|y_{\varepsilon}\right| d \mu\right) \chi_{\Delta_{1}}\right\|_{E}-\frac{\phi(a / 2)}{\phi(a)} \\
& \leq\left\|y_{\varepsilon}\right\|_{E}-\frac{\phi(a / 2)}{\phi(a)}=1-\frac{\phi(a / 2)}{\phi(a)}=M<1 .
\end{aligned}
$$

It follows that for $0<\varepsilon$ small enough it is satisfied that

$$
2-\varepsilon \leq\left\|y_{\varepsilon}\right\|_{E}+M+u(\varepsilon)+v(\varepsilon) \leq 1+M+u(\varepsilon)+v(\varepsilon),
$$

which is a contradiction. Thus

$$
2-\varepsilon \leq 1+\frac{\phi\left(\mu\left(\Delta_{1}\right)\right)}{\phi(a)}-\frac{\phi\left(\mu\left(\Delta_{1}\right)\right)}{\mu\left(\Delta_{1}\right)} \int_{\Delta_{1}}\left|y_{\varepsilon}\right| d \mu+u(\varepsilon)+v(\varepsilon) .
$$

Recall that the function $\phi$ is increasing and $t \mapsto \phi(t) / t$ is decreasing on $(0, \mu(\Omega))$. Therefore and in view of $a / 2 \leq \mu\left(\Delta_{1}\right) \leq a$, we have

$$
0<\frac{\phi(a / 2)}{a} \leq \frac{\phi\left(\mu\left(\Delta_{1}\right)\right)}{\mu\left(\Delta_{1}\right)} \leq 2 \frac{\phi(a)}{a}<\infty .
$$

Thus by (4.6), we have

$$
\lim _{\varepsilon \rightarrow 0} \phi\left(\mu\left(\Delta_{1}\right)\right)=\phi(a) \text { and } \lim _{\varepsilon \rightarrow 0} \int_{\Delta_{1}}\left|y_{\varepsilon}\right| d \mu=0 .
$$

By (4.1) we have $\lim _{\varepsilon \rightarrow 0}\left\|b y_{\varepsilon} \chi_{A}\right\|_{1}=1$, which implies in view of (4.7) and (4.3) that

$$
\lim _{\varepsilon \rightarrow 0} \int_{C_{2}}\left|y_{\varepsilon}\right| d \mu=\frac{1}{b}
$$

so $\mu\left(C_{2}\right)>0$ for $\varepsilon$ small enough. Since $0<\mu\left(C_{2}\right) \leq \mu\left(\Delta_{2}\right) \leq \frac{a}{2} \leq \mu\left(\Delta_{1}\right)$, by also using Lemma 2.3 we have

$$
\begin{aligned}
\frac{\phi\left(\mu\left(\Delta_{1}\right)\right)}{\mu\left(\Delta_{1}\right)} \int_{C_{2}}\left|y_{\varepsilon}\right| d \mu & \leq \frac{\phi\left(\mu\left(C_{2}\right)\right)}{\mu\left(C_{2}\right)} \int_{C_{2}}\left|y_{\varepsilon}\right| d \mu \\
& =\left\|\left(\frac{1}{\mu\left(C_{2}\right)} \int_{C_{2}}\left|y_{\varepsilon}\right| d \mu\right) \chi_{C_{2}}\right\|_{E} \leq\left\|y_{\varepsilon}\right\|_{E}=1 .
\end{aligned}
$$


It follows that $\phi\left(\mu\left(\Delta_{1}\right)\right) \int_{C_{2}}\left|y_{\varepsilon}\right| d \mu \leq \mu\left(\Delta_{1}\right) \leq a$. In view of (4.7) and (4.8) we have that $\lim _{\varepsilon \rightarrow 0} \phi\left(\mu\left(\Delta_{1}\right)\right) \int_{C_{2}}\left|y_{\varepsilon}\right| d \mu=\frac{\phi(a)}{b}=a$. As a consequence, we get $\lim _{\varepsilon \rightarrow 0} \mu\left(\Delta_{1}\right)=a$, and thus

$$
\lim _{\varepsilon \rightarrow 0} \mu\left(\Delta_{2}\right)=\lim _{\varepsilon \rightarrow 0} \mu\left(C_{2}\right)=0 .
$$

Again by (4.7) and (4.8), we have that $\lim _{\varepsilon \rightarrow 0} \frac{\phi\left(\mu\left(\Delta_{1}\right)\right)}{\mu\left(\Delta_{1}\right)} \int_{C_{2}}\left|y_{\varepsilon}\right| d \mu=1$. Therefore by (4.9) we get

$$
\lim _{\varepsilon \rightarrow 0} \frac{\phi\left(\mu\left(C_{2}\right)\right)}{\mu\left(C_{2}\right)}=\frac{\phi(a)}{a} .
$$

Thus $\lim _{s \rightarrow 0} \frac{\phi(s)}{s}=\frac{\phi(a)}{a}$. Taking $a=1$ we get $\lim _{s \rightarrow 0} \frac{\phi(s)}{s}=1$. Hence for every $0 \leq t \leq \mu(\Omega), \phi(t) / t=1$, and so $\phi(t)=t$, which completes the proof in view of Lemma 2.2 .

In 2] a partial characterization of the Daugavet property in an Orlicz space $L_{\varphi}$ was given. Now applying Theorem 4.1 we give a complete characterization of Orlicz spaces endowed with the Luxemburg norm having the Daugavet property. We refer to [2] for definitions and notation on Orlicz spaces. First we show the following lemma.

Lemma 4.2. Let $(\Omega, \mathcal{S}, \mu)$ be an infinite atomless measure space. If an Orlicz

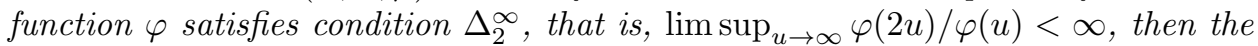
Orlicz space $L_{\varphi}$ equipped with the Luxemburg norm satisfies conditions (i) - (iii) of Theorem 4.1 .

Proof. Let $\|\cdot\|_{\varphi}$ be the Luxemburg norm in $L_{\varphi}$. By $\Delta_{2}^{\infty}$ condition, the space $L_{\varphi}(A)$ is order continuous for any $A \in \mathcal{S}$ with $\mu(A)<\infty$. It is also well known that $L_{\varphi}$ satisfies the Fatou property and so is fully symmetric.

We claim that condition (iii) of Theorem 4.1 is satisfied. Let $A \in \mathcal{S}$ with $\mu(A)<$ $\infty$ and let for all $n \in \mathbb{N}, x, y_{n} \in S_{L_{\varphi}}$ be such that $\left\|y_{n} \chi_{A}\right\|_{\varphi} \rightarrow 1$ and $\left\|x+y_{n}\right\|_{\varphi} \rightarrow 2$.

Denote $I_{\varphi}(z)=\int_{\Omega} \varphi(|z|) d \mu$ for any $z \in L^{0}$. It is well known that if $\mu$ is atomless and $\varphi$ satisfies condition $\Delta_{2}^{\infty}$ and $\mu(A)<\infty$, then $\left\|y_{n} \chi_{A}\right\|_{\varphi} \rightarrow 1$ implies that $I_{\varphi}\left(y_{n} \chi_{A}\right) \rightarrow 1$ (see for instance [4, Theorem 1.39]). Hence $1=\left\|y_{n}\right\|_{\varphi} \geq I_{\varphi}\left(y_{n}\right) \rightarrow 1$, and thus

$$
I_{\varphi}\left(y_{n} \chi_{A^{c}}\right)=I_{\varphi}\left(y_{n}\right)-I_{\varphi}\left(y_{n} \chi_{A}\right) \rightarrow 0 .
$$

Let $1<\alpha_{n}<\left\|x+y_{n}\right\|_{\varphi}$ and $\alpha_{n} \rightarrow 2$. Then $\left\|\frac{x+y_{n}}{\alpha_{n}}\right\|_{\varphi}>1$ and hence $I_{\varphi}\left(\frac{x+y_{n}}{\alpha_{n}}\right)>$ 1. Notice that the modular $I_{\varphi}\left(\frac{x+y_{n}}{\alpha_{n}}\right)$ could be equal to $\infty$. We also have that $I_{\varphi}\left(\frac{y_{n} \chi_{A^{c}}}{\alpha_{n}}\right) \leq \frac{1}{\alpha_{n}} I_{\varphi}\left(y_{n} \chi_{A^{c}}\right)<1$. Hence

$$
I_{\varphi}\left(\frac{x+y_{n} \chi_{A}}{\alpha_{n}}\right) \geq 1-I_{\varphi}\left(\frac{y_{n} \chi_{A^{c}}}{\alpha_{n}}\right) \geq 1-\frac{1}{\alpha_{n}} I_{\varphi}\left(y_{n} \chi_{A^{c}}\right):=\gamma_{n} .
$$

Clearly $0<\gamma_{n} \leq 1$ and $\gamma_{n} \rightarrow 1$. Thus

$$
I_{\varphi}\left(\frac{x+y_{n} \chi_{A}}{\gamma_{n} \alpha_{n}}\right) \geq \frac{1}{\gamma_{n}} I_{\varphi}\left(\frac{x+y_{n} \chi_{A}}{\alpha_{n}}\right) \geq 1 .
$$

Hence $\left\|x+y_{n} \chi_{A}\right\|_{\varphi} \geq \gamma_{n} \alpha_{n}$, and so $\left\|x+y_{n} \chi_{A}\right\|_{\varphi} \rightarrow 2$ as $n \rightarrow \infty$, which proves the claim and completes the proof. 
Corollary 4.3. Let $L_{\varphi}$ be an Orlicz space equipped with the Luxemburg norm over atomless measure space $(\Omega, \mathcal{S}, \mu)$. If $L_{\varphi}$ has the Daugavet property, then $L_{\varphi}$ is isometrically isomorphic to $L_{1}$.

Proof. If $\mu(\Omega)<\infty$, then we apply Theorem 3.6. Otherwise, if $L_{\varphi}$ has the Daugavet property, in view of [2, Theorem 2.1] we have that $\lim _{u \rightarrow \infty} \frac{\varphi(u)}{u}<\infty$. It follows by convexity of $\varphi$ that for some $u_{0} \geq 1, K>0$, it holds that $\varphi(1) u \leq \varphi(u) \leq K u$ for all $u \geq u_{0}$. Hence $\varphi$ satisfies condition $\Delta_{2}^{\infty}$. We finish the proof by applying Lemma 4.2 and Theorem 4.1 .

Recall that a Banach lattice $(X,\|\cdot\|)$ is said to be uniformly monotone $(X \in$ $(U M)$ for short) if for any $\varepsilon>0$ there exists $\delta(\varepsilon)>0$ such that for all $0 \leq y \leq x$ with $\|x\|=1$ and $\|y\| \geq \varepsilon$, it holds that $\|x-y\| \leq 1-\delta(\varepsilon)$.

It is clear that a Banach lattice $X$ is uniformly monotone whenever for any $0 \leq y_{n} \leq x_{n} \in X,\left\|x_{n}\right\|=1$, if $\left\|x_{n}-y_{n}\right\| \rightarrow 1$, then $\left\|y_{n}\right\| \rightarrow 0$. It is well known that a uniformly monotone Banach lattice is order continuous [14, Proposition 2.2].

Theorem 4.4. A uniformly monotone r.i. space E over an infinite atomless measure space $(\Omega, \mathcal{S}, \mu)$ with the Daugavet property is isometrically isomorphic to $L_{1}$.

Proof. We will show that the assumptions (i)-(iii) of Theorem 4.1 are satisfied. Notice that any uniformly monotone space is order continuous, and thus $E(A)$ is order continuous for any $A \in \mathcal{S}$ and also $E$ is fully symmetric 3. Now let $A \in \mathcal{S}$ with $\mu(A)<\infty, x, y_{\varepsilon} \in S_{E}$ for $\varepsilon>0,\left\|y_{\varepsilon} \chi_{A}\right\|_{E} \rightarrow 1$ and $\left\|x+y_{\varepsilon}\right\|_{E} \rightarrow 2$ as $\varepsilon \rightarrow 0$. Then by uniform monotonicity $\left\|y_{\varepsilon} \chi_{A^{c}}\right\|_{E} \rightarrow 0$ as $\varepsilon \rightarrow 0$, and so $\left\|x+y_{\varepsilon} \chi_{A}\right\|_{E} \rightarrow$ 2 .

Remark 4.5. It is worth noticing that property (iii) in Theorem4.1 is not equivalent to (UM) of E. Clearly (UM) is stronger than (iii). We observe that in Orlicz spaces over infinite atomless measure space they are not equivalent. In fact, it is well known that Orlicz space $L_{\varphi}$ is uniformly monotone if and only if it is order continuous [13], which is equivalent to the fact that $\varphi$ satisfies condition $\Delta_{2}$ for all arguments. However, in Lemma 4.2 we showed that the weaker condition $\Delta_{2}^{\infty}$ is sufficient for (iii). In that perspective condition (iii) may be viewed as a sort of uniform monotonicity for "large arguments".

We will show further that under some conditions on the fundamental function of r.i. space $E$, if $E=L_{1}$ as sets and $E$ has the Daugavet property, then $E$ is isometrically equivalent to $L_{1}$. We start with some technical lemma.

Lemma 4.6. Let $E$ be an r.i. space with the weak Fatou property and such that $E \subset L_{1}$. Then there is a norm $\|\cdot\|_{0}$ on $E$ such that $\left(E,\|\cdot\|_{0}\right)$ is an r.i. space isometric to $E$ and also satisfies $\sup _{t \in(0, \mu(\Omega))} \frac{t}{\psi(t)}=1$ where $\psi(t)=\left\|\chi_{(0, t)}\right\|_{0}$. Consequently, $\left(E,\|\cdot\|_{0}\right) \hookrightarrow L_{1}$ with the embedding constant equal to one.

Proof. Let $c=\|$ id: $E \rightarrow L_{1} \|$ and $\|f\|_{0}=c\|f\|_{E}$. Then $\|f\|_{1} \leq\|f\|_{0}$, and so $t \leq \psi(t)$ for all $0 \leq t \leq \mu(\Omega)$. Suppose now that $t \leq \alpha \psi(t)$ for all $0 \leq t \leq \mu(\Omega)$ and some $0<\alpha<1$. Then following the proof of Lemma 2.2 we get $\|f\|_{1} \leq \alpha\|f\|_{0}$ for all $f \in E$, which contradicts the choice of $c$.

Remark 4.7. Notice that if $E$ is an r.i. separable space with the Fatou property and has the Daugavet property, then it follows by [2, Proposition 2.5] that $L_{1} \hookrightarrow E$. If in addition we assume that $E \subset L_{1}$, then by Lemma 4.6 we can find a new norm 
$\|\cdot\|_{0}$ in $E$ such that $E_{0}=\left(E,\|\cdot\|_{0}\right)$ is an r.i. space and $\|$ id: $E_{0} \rightarrow L_{1} \|=1$. Hence, we get that there exists $C>0$ such that for all $x \in E$,

$$
\|x\|_{1} \leq\|x\|_{0} \leq C\|x\|_{1} \quad \text { and } \quad \sup _{t \in(0, \mu(\Omega))} \frac{t}{\phi_{E_{0}}(t)}=1 .
$$

We prove below that under the additional assumption that the supremum in the above formula is attained, if $E$ has the Daugavet property, then $E_{0}=L_{1}$ endowed with its canonical norm.

Theorem 4.8. Let $E$ be an r.i. space over infinite atomless measure space $(\Omega, \mathcal{S}, \mu)$ with the Fatou property. Assume also that

(1) $s \leq \phi_{E}(s)$ for every $0<s<\mu(\Omega)$,

(2) $\phi_{E}(t)=t$ for some $t>0$.

If $E$ is separable and has the Daugavet property, then $E=L_{1}$ isometrically.

Proof. Assume that $\phi_{E}(1)=1$ and follow the proof of Theorem 4.4. There exists $A \subset \Omega$ such that $\mu(A)=t$. Letting $x=\frac{1}{\phi_{E}(t)} \chi_{A}$ and $y=-\frac{\phi_{E}(t)}{t} \chi_{A}=-\chi_{A}$, we have that $x \in S_{E}$ and $y \in S_{E^{\prime}}$. Let $F$ be an integral functional induced by $y$. By the Daugavet property there exists $y_{\varepsilon} \in S_{E}$ such that

$$
F\left(y_{\varepsilon}\right) \geq 1-\varepsilon \quad \text { and } \quad\left\|x+y_{\varepsilon}\right\|_{E}>2-\varepsilon .
$$

Our hypothesis (1) in combination with Lemma 2.2 yields $\|z\|_{1} \leq\|z\|_{E}$ for all $z \in E$. We have

$$
1-\varepsilon \leq \int_{A} y_{\varepsilon} d \mu \leq \int_{\Omega}\left|y_{\varepsilon} \chi_{A}\right| d \mu \leq\left\|y_{\varepsilon} \chi_{A}\right\|_{E} \leq\left\|y_{\varepsilon}\right\|_{E}=1 .
$$

Hence $1=\left\|y_{\varepsilon}\right\|_{E} \geq \int_{\Omega}\left|y_{\varepsilon}\right| d \mu \geq 1-\varepsilon+\int_{A^{c}}\left|y_{\varepsilon}\right| d \mu$, and so $\left\|y_{\varepsilon} \chi_{A^{c}}\right\|_{1} \leq \varepsilon$. Now by [2, Proposition 2.5], it follows that $L_{1} \hookrightarrow E$, and so

$$
\left\|y_{\varepsilon} \chi_{A^{c}}\right\|_{E} \rightarrow 0 \quad \text { as } \quad \varepsilon \rightarrow 0
$$

Thus we can assume further that

$$
\left\|x+y_{\varepsilon} \chi_{A}\right\|_{E}>2-\varepsilon .
$$

Now following the proof of Theorem 4.4 from (4.2) we obtain that $\lim _{s \rightarrow 0} \frac{\phi_{E}(s)}{s}=1$, which implies that $\phi_{E}(s)=s$ for all $0 \leq s \leq \mu(\Omega)$. Consequently, $\stackrel{s}{E}=L_{1}$ isometrically by Lemma 2.2 .

In general, the Daugavet property is not inherited by 1-complemented subspaces. For instance, $L_{1}[0,1]$ has the Daugavet property but $\ell_{1}$ does not have it. The following result shows that in the case of uniformly monotone Banach lattices with the Daugavet property the following natural subspaces inherit the property.

Theorem 4.9. Let $E$ be a uniformly monotone Banach lattice over a measure space $(\Omega, \mathcal{S}, \mu)$. If $E$ has the Daugavet property, then $E(A)$ also has this property for every $A \in \mathcal{S}$ with $0<\mu(A) \leq \mu(\Omega)$, where $E(A)=\left\{x \chi_{A}: x \in E\right\}$.

Proof. Let $R: E(A) \rightarrow E(A)$ be a nonnull finite rank operator. Denote by $P: E \rightarrow$ $E(A)$ the linear projection given by

$$
P x=x \chi_{A}, \quad x \in E,
$$

that clearly has norm one. We define the operator $T: E \rightarrow E$ by

$$
T x=R(P x), \quad x \in E .
$$


It is clear that $T$ is a finite rank operator and also $\|T\|=\|R\|$. Our hypothesis $E \in(D P)$ implies that

$$
\|I+T\|=1+\|T\|=1+\|R\| .
$$

Hence there is a sequence $\left(x_{n}\right)$ in $S_{E}$ such that

$$
\left\|x_{n}+T\left(x_{n}\right)\right\|_{E} \rightarrow 1+\|R\| .
$$

As a consequence, we have that

$$
\begin{aligned}
1+\|R\| & =\lim _{n \rightarrow \infty}\left\|x_{n}+R\left(x_{n} \chi_{A}\right)\right\|_{E} \leq \liminf _{n \rightarrow \infty}\left(1+\|R\|\left\|x_{n} \chi_{A}\right\|_{E}\right) \\
& \leq \limsup _{n \rightarrow \infty}\left(1+\|R\|\left\|x_{n} \chi_{A}\right\|_{E}\right) \leq 1+\|R\| .
\end{aligned}
$$

Combining, we conclude by $R \neq 0$ that $\lim _{n \rightarrow \infty}\left\|x_{n} \chi_{A}\right\|_{E}=1$. Since

$$
0 \leq\left|x_{n} \chi_{\Omega \backslash A}\right| \leq\left|x_{n}\right|, \quad\left\|x_{n}\right\|_{E}=1,
$$

and $\left|x_{n}\right|-\left|x_{n} \chi_{\Omega \backslash A}\right|=\left|x_{n} \chi_{A}\right|$ for each $n \in \mathbb{N}$, we deduce by $E \in(U M)$ that $\left\|x_{n} \chi_{\Omega \backslash A}\right\|_{E} \rightarrow 0$. This implies

$$
1+\|R\|=\lim _{n \rightarrow \infty}\left\|x_{n}+R\left(x_{n} \chi_{A}\right)\right\|_{E}=\lim _{n \rightarrow \infty}\left\|x_{n} \chi_{A}+R\left(x_{n} \chi_{A}\right)\right\|_{E} .
$$

Since $x_{n} \chi_{A} \in B_{E(A)}$ for each $n$, we have

$$
\|I+R\|=1+\|R\|
$$

which completes the proof.

We note that the present paper and the results from [2] suggest the conjecture that the only r.i. spaces on infinite atomless measure spaces with the Daugavet property are those which are naturally isometric either to $L_{1}$ or $L_{\infty}$ endowed with their canonical norms. In fact the proofs of the main results of the present paper would give a positive answer provided that the Daugavet property of r.i. spaces over an infinite atomless measure space $(\Omega, \mathcal{S}, \mu)$ would be inherited by $E(A)$ for every $A \in \mathcal{S}$ with positive and finite measure. Notice also that in the class of r.i. $K B$ spaces the conjecture would be true if the uniform monotonicity was a necessary condition for the Daugavet property.

\section{REFERENCES}

[1] Y. A. Abramovich and C. D. Aliprantis, An invitation to operator theory, Graduate Studies in Mathematics, vol. 50, American Mathematical Society, Providence, RI, 2002. MR.1921782 (2003h:47072)

[2] M. D. Acosta, A. Kamińska, and M. Mastyło, The Daugavet property and weak neighborhoods in Banach lattices, J. Convex Anal. 19 (2012), no. 3, 875-912. MR3013764

[3] Colin Bennett and Robert Sharpley, Interpolation of operators, Pure and Applied Mathematics, vol. 129, Academic Press Inc., Boston, MA, 1988. MR.928802 (89e:46001)

[4] Shutao Chen, Geometry of Orlicz spaces, Dissertationes Math. (Rozprawy Mat.) 356 (1996), 204. With a preface by Julian Musielak. MR,1410390 (97i:46051)

[5] I. K. Daugavet, A property of completely continuous operators in the space $C$ (Russian), Uspehi Mat. Nauk 18 (1963), no. 5 (113), 157-158. MR.0157225 (28 \#461)

[6] H. Hudzik, A. Kamińska, and M. Mastyło, Monotonicity and rotundity properties in Banach lattices, Rocky Mountain J. Math. 30 (2000), no. 3, 933-950, DOI 10.1216/rmjm/1021477253. MR.1797824(2002c:46039)

[7] Yevgen Ivakhno, Vladimir Kadets, and Dirk Werner, The Daugavet property for spaces of Lipschitz functions, Math. Scand. 101 (2007), no. 2, 261-279. MR2379289(2009c:46014) 
[8] Vladimir Kadets, Miguel Martín, Javier Merí, and Dirk Werner, Lushness, numerical index 1 and the Daugavet property in rearrangement invariant spaces, Canad. J. Math. 65 (2013), no. 2, 331-348, DOI 10.4153/CJM-2011-096-2. MR3028566

[9] Vladimir M. Kadets, Roman V. Shvidkoy, Gleb G. Sirotkin, and Dirk Werner, Banach spaces with the Daugavet property, Trans. Amer. Math. Soc. 352 (2000), no. 2, 855-873, DOI 10.1090/S0002-9947-99-02377-6. MR.1621757 (2000c:46023)

[10] Anna Kamińska and Mieczysław Mastyło, The Dunford-Pettis property for symmetric spaces, Canad. J. Math. 52 (2000), no. 4, 789-803, DOI 10.4153/CJM-2000-033-9. MR.1767402 (2001g:46062)

[11] L. V. Kantorovich and G. P. Akilov, Functional analysis, 2nd ed., Pergamon Press, Oxford, 1982. Translated from the Russian by Howard L. Silcock. MR664597 (83h:46002)

[12] S. G. Kreı̆n, Yu. İ. Petunīn, and E. M. Semënov, Interpolation of linear operators, Translations of Mathematical Monographs, vol. 54, American Mathematical Society, Providence, R.I., 1982. Translated from the Russian by J. Szücs. MR649411 (84j:46103)

[13] W. Kurc, Strictly and uniformly monotone Musielak-Orlicz spaces and applications to best approximation, J. Approx. Theory 69 (1992), no. 2, 173-187, DOI 10.1016/00219045(92)90141-A. MR.1160253 (93g:46028)

[14] Han Ju Lee, Monotonicity and complex convexity in Banach lattices, J. Math. Anal. Appl. 307 (2005), no. 1, 86-101, DOI 10.1016/j.jmaa.2005.01.017. MR2138977 (2005m:46037)

[15] Joram Lindenstrauss and Lior Tzafriri, Classical Banach spaces. II, Ergebnisse der Mathematik und ihrer Grenzgebiete [Results in Mathematics and Related Areas], vol. 97, SpringerVerlag, Berlin, 1979. Function spaces. MR540367 (81c:46001)

[16] Dirk Werner, Recent progress on the Daugavet property, Irish Math. Soc. Bull. 46 (2001), 77-97. MR.1856978(2002i:46014)

[17] P. Wojtaszczyk, Some remarks on the Daugavet equation, Proc. Amer. Math. Soc. 115 (1992), no. 4, 1047-1052, DOI 10.2307/2159353. MR1126202 (92k:47041)

Departamento de Análisis Matemático, Universidad de Granada, 18071 Granada, SPAIN

E-mail address: dacosta@ugr.es

Department of Mathematical Sciences, The University of Memphis, Memphis, TenNESSEE 38152

E-mail address: kaminska@memphis.edu

Faculty of Mathematics and Computer Science, A. Mickiewicz University and Institute of Mathematics, Polish Academy of Sciences (Poznań branch), Umultowska 87, 61-614 Poznań, Poland

E-mail address: mastylo@amu.edu.pl 